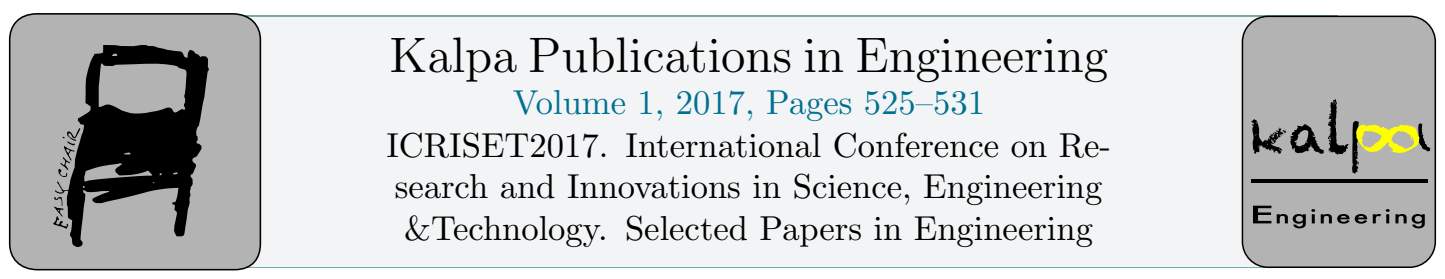

\title{
Performance Evaluation of Different STBC Technique in MIMO System with Rayleigh Fading Environment
}

\author{
Darshankumar C. Dalwadi ${ }^{1}$ \\ ${ }^{1}$ Gujarat Technological University, Ahmedabad, Gujarat, India \\ darshan.dalwadi@bvmengineering.ac.in
}

\begin{abstract}
In this paper, I have presented the performance evaluation of different STBC technique in MIMO Rayleigh fading environment. I have evaluated the BER of SISO, MISO and MIMO technique. I have proved that the lowest value of BER is achieved in STBC technique. I have evaluated the BER of real STBC and complex STBC technique with Rayleigh fading channel. The STBC technique is also reduced the complexity of the receiver. We have also compared the performance of MIMO technique with other techniques like SISO and MISO. The lowest value of BER is achieved in MIMO technique. The STBC technique is exploit the diversity gain by inserting more number of antennas at transmitter side. Simulation results are based on MATLAB software.
\end{abstract}

\section{Introduction}

There are two types of multiple antenna techniques: (1) Spatial multiplexing techniques and (2) Diversity techniques. In this paper, we have presented the concepts of transmitter diversity techniques which improve the BER performance by increasing the number antennas at the transmitter side. We have considered the Rayleigh fading wireless channel [1,2]. For a Rayleigh fading wireless channel the BER value is higher than AWGN reference channel. Due to this the performance over the Rayleigh fading wireless channel is significantly poor, even at high SNR. The main objective of the transmitter diversity techniques is to reduce the value of the BER as SNR increases in the Rayleigh fading environment. We have compared the performance of MIMO system with SISO and MISO system [3]. 
Practically transmitter diversity is preferred because it is feasible to include more number of antennas at transmitter or base station side. Transmitter diversity is also reduced the complexity of the receiver [4]. In the downlink it is important to exploit diversity at the transmitter side. To implement this phenomenon, we have two different transmission techniques. (1) Transmit Beamforming: In this technique CSI is available at both transmitter and receiver side. This technique provides both diversity gain and antenna array gain. (2) Space Time Block Coding (STBC): In this technique, CSI is available only at the receiver side. In this technique we have achieved fully diversity gain but we cannot achieve antenna array gain. Again code rate is also less than one if more than two antennas are used at the transmitter. For a special case of STBC, Alamouti transmit diversity, code rate of one can be achieved. In this paper, we have focused on STBC techniques [5].

\section{Block Diagram of Space Time Block Coding}

\subsection{Examples of different antenna techniques}

Figure 1 shows the different types of antenna techniques: Some examples are SIMO, MISO, and MIMO techniques [6].

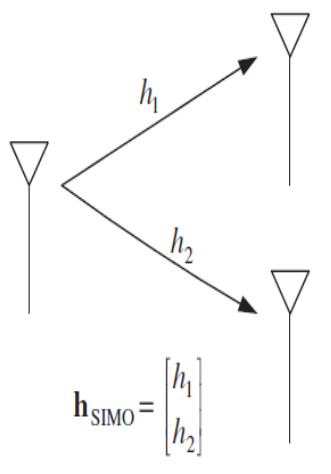

(a) SIMO

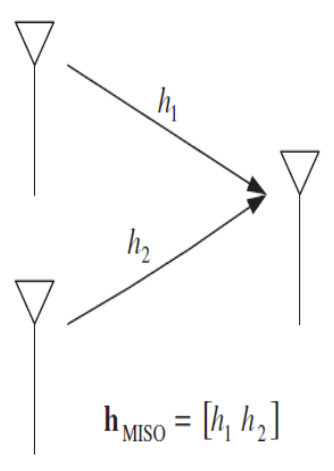

(b) MISO

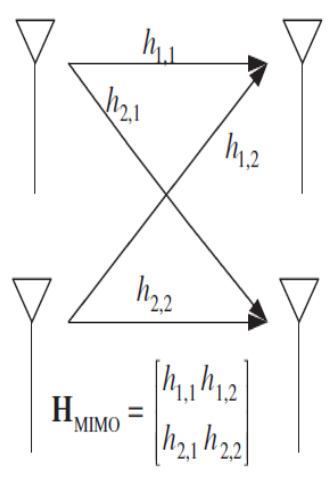

(c) MIMO

Figure 1: Different types of antenna techniques

\subsection{System Model of Space Time Block Coding (STBC)}

Figure 2 shows the block diagram of STBC MIMO systems with $\mathrm{N}_{T}$ transmit antennas and $\mathrm{N}_{R}$ receive antennas. 


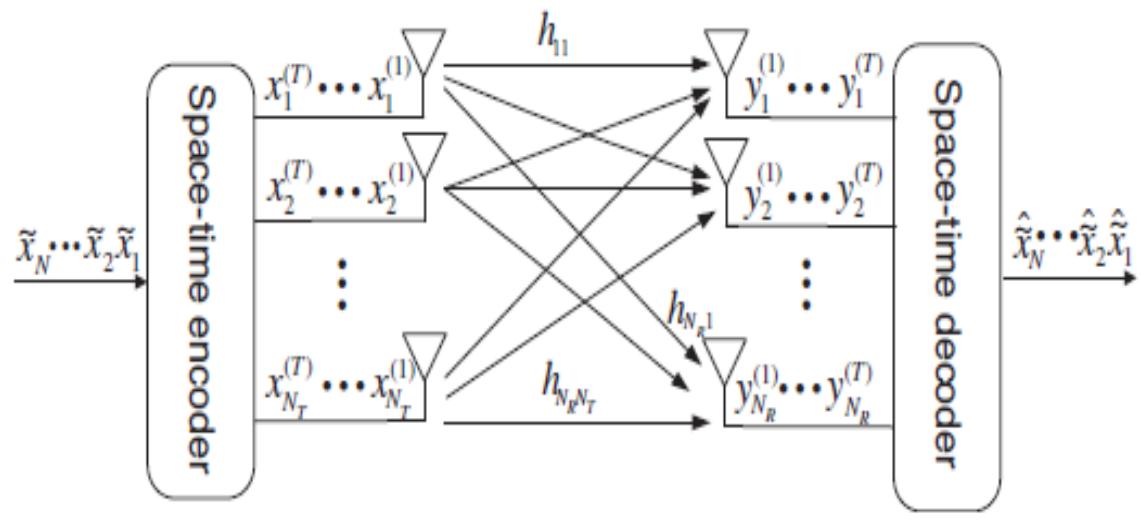

Figure 2: System Model of STBC MIMO systems

In the STBC-MIMO systems, bit stream is mapped into symbol stream $\left\{x_{i}\right\}_{i=1}^{N}$. From, figure 2, the system equation is given as,

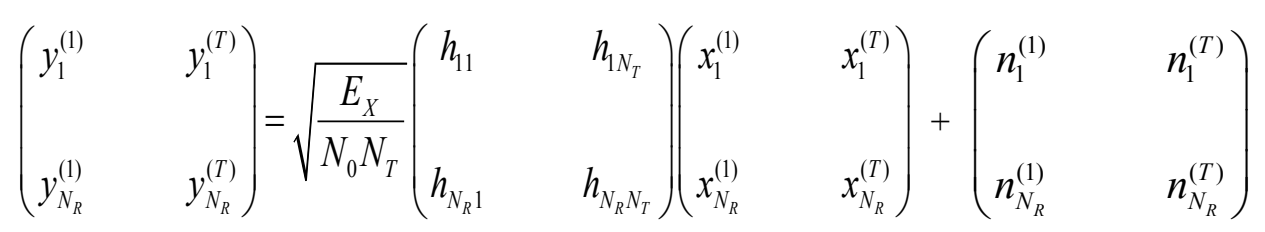

Where, $\mathrm{y}$ represent the output signal, $\mathrm{h}$ represent the system function, $\mathrm{x}$ represent the input signal and $\mathrm{n}$ represent the noise signal.

\section{Different types of Space Time Block Code}

In this section we have discussed the two types of space time block code techniques: (i) Real $\mathrm{STBC}$ and (ii) Complex STBC.

\subsection{Real Space Time Block Code}

This section presents the examples of the STBC technique with real entries. We consider the square STC with the coding rate of 1 and diversity order of $\mathrm{N}_{\mathrm{T}}=2$, 4 . Following are the equations of those real space time codes.

$$
X_{2, \text { real }}=\left(\begin{array}{cc}
x_{1} & -x_{2} \\
x_{2} & x_{1}
\end{array}\right)
$$




$$
\mathbf{X}_{4, \text { real }}=\left[\begin{array}{cccc}
x_{1} & -x_{2} & -x_{3} & -x_{4} \\
x_{2} & x_{1} & x_{4} & -x_{3} \\
x_{3} & -x_{4} & x_{1} & x_{2} \\
x_{4} & x_{3} & -x_{2} & x_{1}
\end{array}\right]
$$

Coding rate is given by,

$$
\mathrm{R}=\mathrm{N} / \mathrm{T}=4 / 4=1 \text {. }
$$

\subsection{Complex Space Time Block Code}

In Alamouti code [7], a complex STBC with $\mathrm{N}_{\mathrm{T}}=2$, which achieves the maximum diversity order of 2 with the maximum possible coding rate $\mathrm{R}=1$, given as,

$$
X_{2, \text { complex }}=\left(\begin{array}{cc}
x_{1} & -x_{2}^{*} \\
x_{2} & x_{1}^{*}
\end{array}\right)
$$

Now, consider the case in which $\mathrm{N}_{\mathrm{T}}=4$. In this case, complex signal is given as,

$$
\mathbf{X}_{4, \text { complex }}=\left[\begin{array}{cccccccc}
x_{1} & -x_{2} & -x_{3} & -x_{4} & x_{1}^{*} & -x_{2}^{*} & -x_{3}^{*} & -x_{4}^{*} \\
x_{2} & x_{1} & x_{4} & -x_{3} & x_{2}^{*} & x_{1}^{*} & x_{4}^{*} & -x_{3}^{*} \\
x_{3} & -x_{4} & x_{1} & x_{2} & x_{3}^{*} & -x_{4}^{*} & x_{1}^{*} & x_{2}^{*} \\
x_{4} & x_{3} & -x_{2} & x_{1} & x_{4}^{*} & x_{3}^{*} & -x_{2}^{*} & x_{1}^{*}
\end{array}\right]
$$

For equation (6), transmits $\mathrm{N}=4$ symbols over $\mathrm{T}=8$ symbol periods, thus yielding a coding rate of $\mathrm{R}=1 / 2$.

\section{Decoder of Space Time Block Code}

Let us first consider a Real STBC technique with $\mathrm{X}_{4 \text {, real }}$ in equation (3). We express the received signal as,

$$
\left[y_{1} y_{2} y_{3} y_{4}\right]=\sqrt{\frac{E_{x}}{4 N_{0}}}\left[h_{1} h_{2} h_{3} h_{4}\right] X_{4, \text { real }}+\left[n_{1} n_{2} n_{3} n_{4}\right]
$$

Where,

$$
\mathbf{X}_{4, \text { real }}=\left[\begin{array}{cccc}
x_{1} & -x_{2} & -x_{3} & -x_{4} \\
x_{2} & x_{1} & x_{4} & -x_{3} \\
x_{3} & -x_{4} & x_{1} & x_{2} \\
x_{4} & x_{3} & -x_{2} & x_{1}
\end{array}\right]
$$

The received signal decision statistics are derived as:

$$
y_{\text {effe }, 1}=\left(h_{e f f e, 1}^{1}\right)^{H} y_{\text {effe }}^{1}=\sqrt{\frac{E_{x}}{3 N_{0}}}\left(\left|h_{1}\right|^{2}+\left|h_{2}\right|^{2}+\left|h_{3}\right|^{2}\right) x_{1}+\left(h_{e f f e, 1}^{1}\right)^{H} n_{e f f e}^{1}
$$




$$
\begin{aligned}
& y_{\text {effe }, 2}=\left(h_{\text {effe }, 2}^{2}\right)^{H} y_{\text {effe }}^{2}=\sqrt{\frac{E_{x}}{3 N_{0}}}\left(\left|h_{1}\right|^{2}+\left|h_{2}\right|^{2}+\left|h_{3}\right|^{2}\right) x_{2}+\left(h_{\text {effe }, 2}^{2}\right)^{H} n_{\text {effe }}^{2} \\
& y_{\text {effe }, 3}=\left(h_{\text {effe, }, 3}^{3}\right)^{H} y_{\text {effe }}^{3}=\sqrt{\frac{E_{x}}{3 N_{0}}}\left(\left|h_{1}\right|^{2}+\left|h_{2}\right|^{2}+\left|h_{3}\right|^{2}\right) x_{3}+\left(h_{\text {effe }, 3}^{3}\right)^{H} n_{\text {effe }}^{3}
\end{aligned}
$$

The Maximum likelihood detection is performed as,

$$
\hat{x}_{i, M L}=Q\left(\frac{y_{\text {effe }, i}}{\sqrt{\frac{E_{x}}{3 N_{0}} \sum_{k=1}^{3}\left|h_{k}\right|^{2}}}\right), i=1,2,3
$$

\section{Simulation Results}

In this section we have discussed the simulation results of BER v/s SNR for different types of transmit antenna diversity techniques like MISO, MIMO with respect to Rayleigh fading wireless channel environment. We have evaluated the BER of QPSK and 16-QAM modulation technique with respect to large number of antennas at transmitter and receiver side.
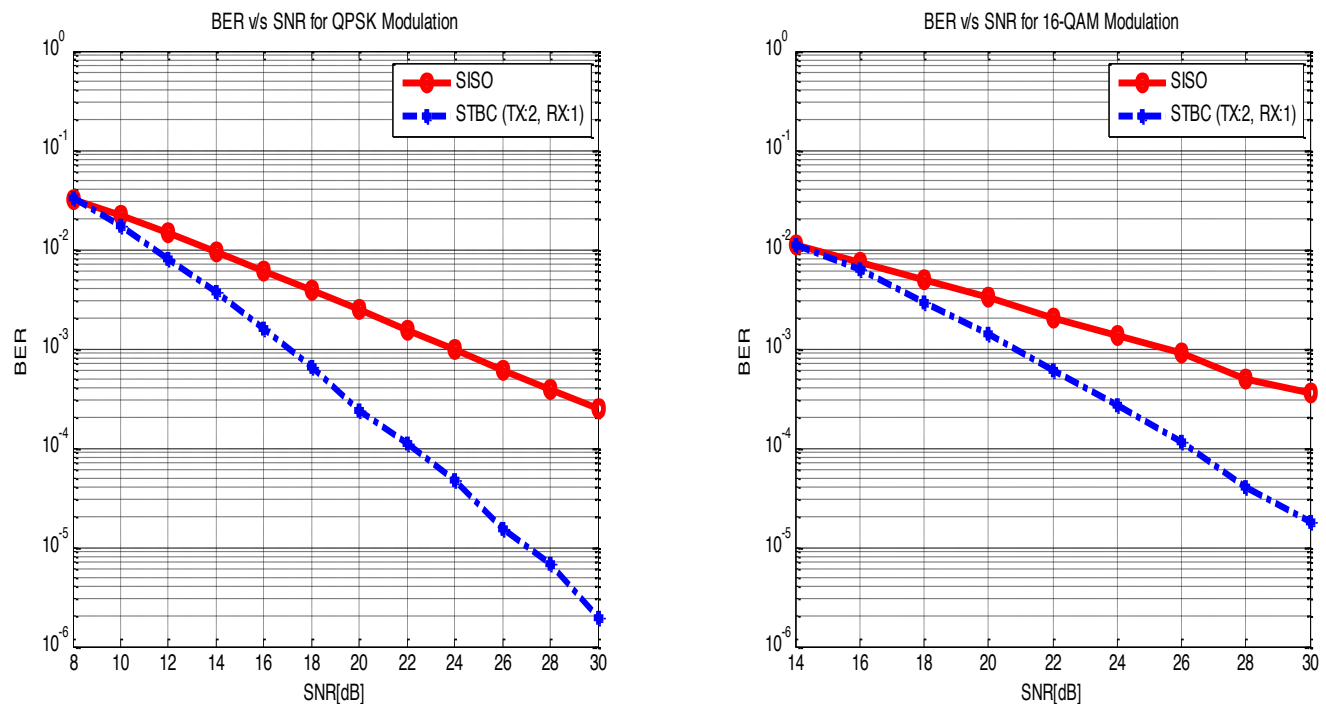

Figure 3: BER v/s SNR for QPSK Modulation with Transmit diversity (STBC)

Figure 4: BER v/s SNR for 16-QAM Modulation with Transmit diversity (STBC) 
Figure 3 shows BER v/s SNR for QPSK modulation technique with STBC. From figure 3, it can be seen that compared to SISO system in STBC coding BER is improved. Lowest value of BER is achieved in STBC technique.

Figure 4 shows the BER v/s SNR for 16-QAM modulation technique with STBC. From figure 4 , it can be seen that compared to SISO system in STBC coding BER is improved. Lowest value of BER is achieved in STBC technique.

Figure 5 shows the BER v/s SNR for QPSK modulation technique with STBC with more number of antennas at transmitter and receiver side. Compared to figure 3, we can say that as we increased the number of antennas at transmitter and receiver side, the BER is reduced.

Figure 6 shows the BER v/s SNR for 16-QAM modulation technique with STBC with more number of antennas at both side. Compared to figure 4, we can say that as we increased the number of antennas at transmitter and receiver side, the BER is reduced. But compared to figure 5, for 16-QAM modulation technique BER is higher than QPSK modulation technique. Because as we increased the $\mathrm{M}$ ary number BER is also increased.
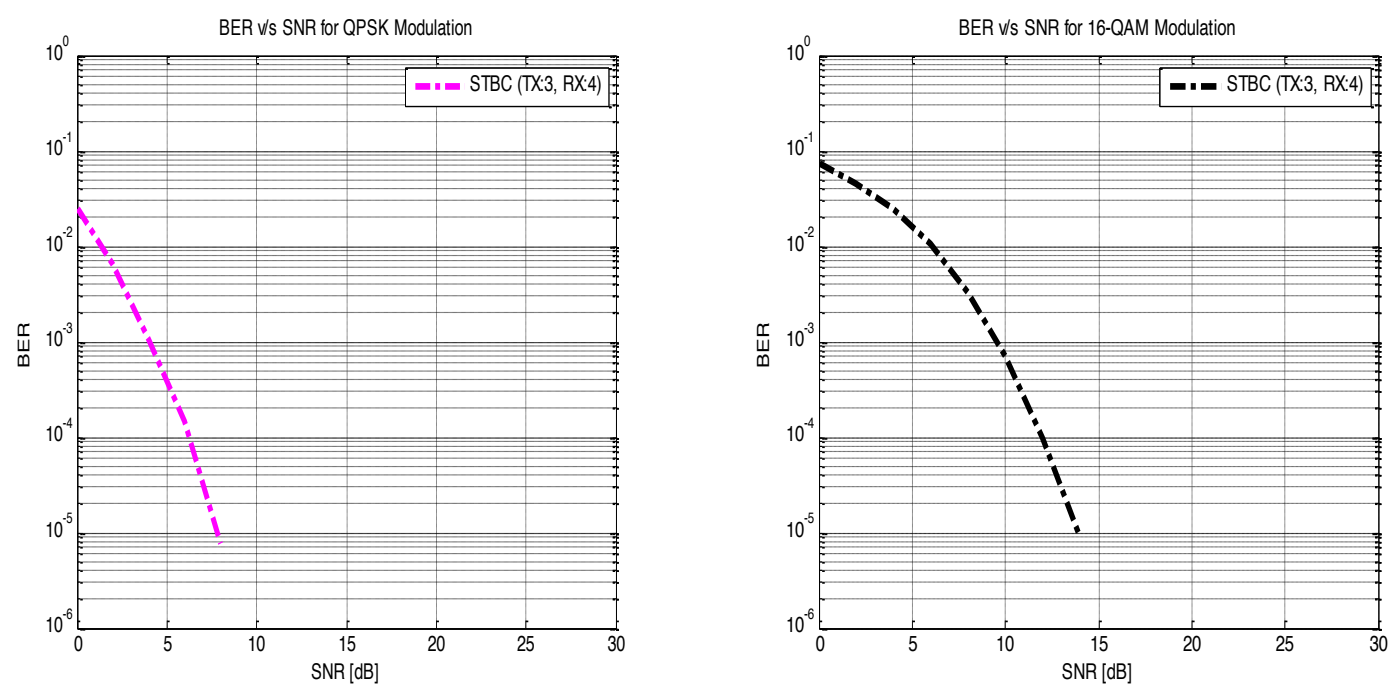

Figure 5: BER v/s SNR for QPSK Modulation with Transmit diversity (STBC) with $\mathrm{TX}=3$ and $\mathrm{RX}=4$ Figure 6: BER v/s SNR for 16-QAM Modulation with Transmit diversity (STBC) with $\mathrm{TX}=3$ and $\mathrm{RX}=4$ 


\section{Conclusion}

From this paper, we conclude that as we include the transmitter diversity that is as we increased the number of antennas at transmitter side the BER is reduced. From simulation results of figure 3 to 6 , we say that as we increased the number of antennas at transmitter side we have achieved the lowest value of BER. We have also concluded that compared to QPSK modulation technique and 16-QAM modulation technique, the lowest value of BER is achieved in QPSK modulation. Because as we increased the M-ary number the BER is also increased. The STBC technique is also reduced the complexity of the receiver by inserting more number of antennas at transmitter side.

\section{References}

[1] Y. Zhang, S. B. Gelfand, M. P. Fitz, "Soft output demodulation on frequency selective Rayleigh fading channels using AR channel models", IEEE transactions on communications vol. 55, No. 10, October 2007.

[2] T. Eng, N. Kong, L. B. Milstein, "Comparison of Diversity combining Techniques for Rayleigh fading channels", IEEE transactions on communications vol. 44, No. 9, September 1996.

[3] S. M. Alamouti, "A simple transmit diversity techniques for wireless communications", IEEE J. selected Areas Communications, vol. 16, pp. 1451-1458, October 1998.

[4] D. C. Dalwadi, H. B. Soni, "A novel channel estimation technique of MIMO-OFDM system based on Modified Kalman filter", Indian journal of science and technology, vol. 9, September 2016.

[5] J. Wu, G. J. Saulnier, "Orthogonal space time block code over time varying flat fading channels: channel estimation, detection and performance analysis", IEEE transactions on communication, vol. 55, no. 5, pp. 1077-1087, May 2007.

[6] P. A. Dighe, R. K. Mallik, S. S. Jamuar, "Analysis of transmit-receiver diversity in Rayleigh fading", IEEE transactions on communications, vol. 51, no. 4, pp. 694-703, April 2003.

[7] Y. N. Trivedi, A. K. Chaturvedi, "Performance analysis of multiple input single output systems using transmit beamforming and antenna selection with delayed channel state information at the transmitter", IET communications, vol. 5, issue 6, pp. 827834, 2011.

[8] A. J. Goldsmith and S. G. Chua, "Variable-rate variable power M-QAM for fading channel", IEEE transactions on Communications, vol. 45, No. 10, pp. 1218-1230, October 1997.

[9] C. Shannon, "A mathematical theory of communication”, Bell labs technical journal, 379-423 and 623-656, July and October 1948.

[10] D. A. Gore, R. W. Heath and A. J. Paulraj, “Transmit selection in Spatial multiplexing system”, IEEE communication letters, vol. 6, No. 11, pp. 491-493, November 2002. 